This item was submitted to Loughborough's Research Repository by the author.

Items in Figshare are protected by copyright, with all rights reserved, unless otherwise indicated.

\title{
Evaluation of thin, flexible sensors for time-resolved grip force measurement
}

PLEASE CITE THE PUBLISHED VERSION

PUBLISHER

Professional Engineering Publishing / @ IMechE

LICENCE

CC BY-NC-ND 4.0

\section{REPOSITORY RECORD}

Komi, Erin R., Jonathan R. Roberts, and Steve Rothberg. 2019. "Evaluation of Thin, Flexible Sensors for Time-resolved Grip Force Measurement”. figshare. https://hdl.handle.net/2134/3578. 
This item was submitted to Loughborough's Institutional Repository by the author and is made available under the following Creative Commons Licence conditions.

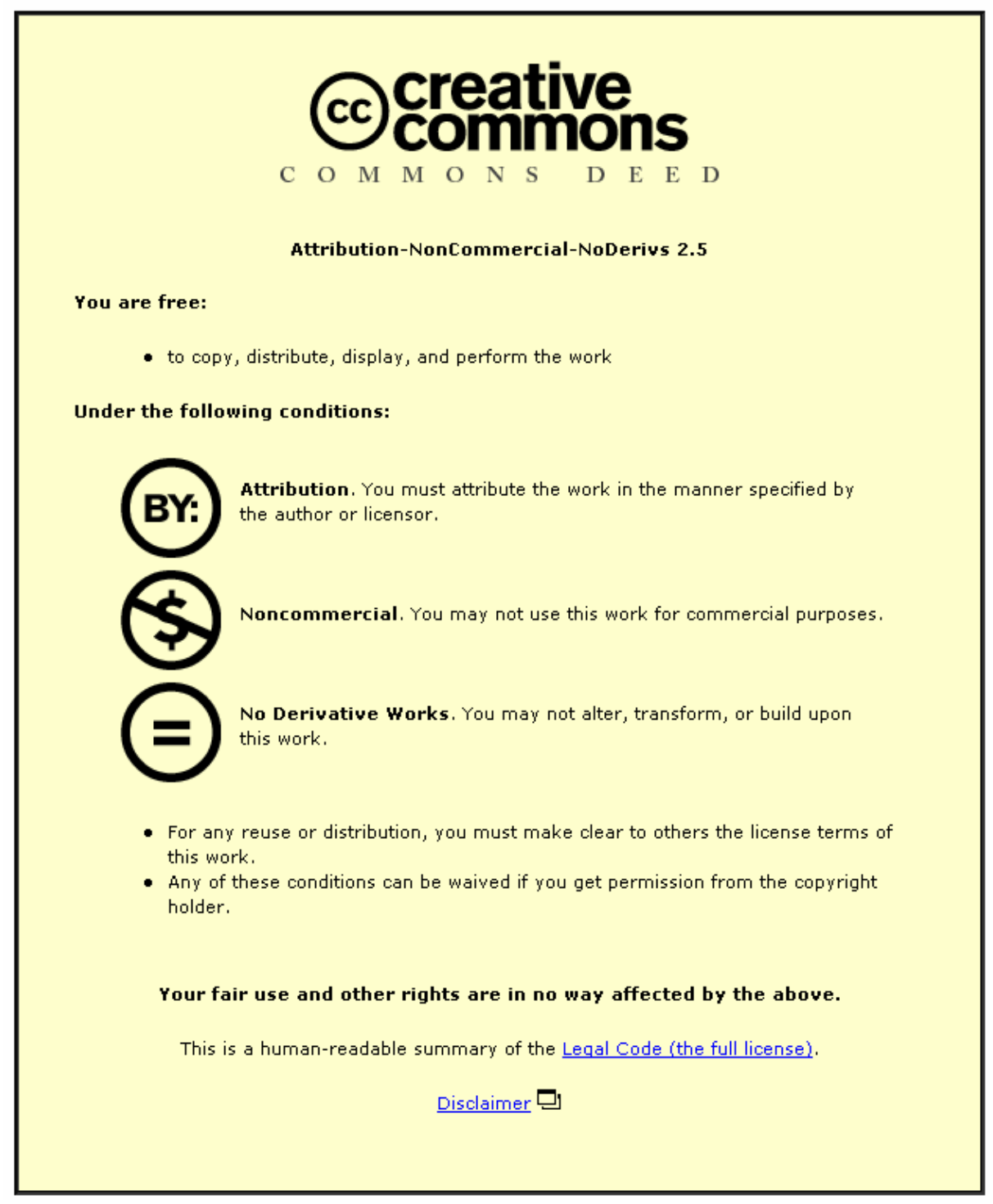

For the full text of this licence, please go to: http://creativecommons.org/licenses/by-nc-nd/2.5/ 


\title{
Evaluation of thin, flexible sensors for time-resolved grip force measurement
}

\author{
E R Komi* , J R Roberts, and S J Rothberg \\ Mechanical and Manufacturing Engineering, Loughborough University, Loughborough, UK
}

The manuscript was received on 5 April 2007 and was accepted after revision for publication on 3 August 2007.

DOI: 10.1243/09544062JMES700

\begin{abstract}
Three types of thin, flexible force sensor were studied under a variety of loading conditions to determine their suitability for measuring grip force. Static accuracy, hysteresis, repeatability, and drift errors were established, the effects of shear force and surface curvature were considered, and dynamic accuracy and drift were measured. Novel tests were developed to consider dynamic accuracy and sensitivity to shear loadings. Additionally, three sensors were evaluated in a real-life gripping scenario, measuring grip force during a golf shot. Comments are made on sensor performance, ease of use, and durability.
\end{abstract}

Keywords: load cell, pressure, hand, golf, accuracy

\section{INTRODUCTION}

Grip force is measured in clinical and research settings for a host of reasons. In such studies, it is typical for real-life gripping scenarios to be recreated to investigate entities such as maximum grip force, and the effects of loading on vibration transmission and wrist range of motion. The requirement to reproduce representative grip conditions means that the force sensor used must not significantly alter the performance characteristics of the device that is being grasped or the operator's ability to use the device.

Force sensors are available in numerous formats, many of which are well suited to the cylindrical shape of a typical handle. Load cells are commonly used in hand dynamometers where force is measured in one direction, and miniature load cells or simply supported beams with strain gauges have been used to measure force at several locations simultaneously. These methods of force measurement, however, have limitations for grip force measurement. Spatial resolution tends to be poor as sensor size and rigidity make it difficult to apply multiple sensors to curved gripping surfaces. Consequently, while load cells tend to be the most accurate and reliable force sensors available, it

${ }^{*}$ Corresponding author: Mechanical and Manufacturing Engineering, Loughborough University, Loughborough LE11 3TU, UK. email:E.Komi@lboro.ac.uk is not always feasible to use them when monitoring realistic gripping conditions [1-7].

Thin, flexible force sensors have appeared as an alternative for many force measurement applications where load cells are not appropriate. These sensors fit easily over curved surfaces, are extremely light weight and are available in a wide variety of formats. For measurement of grip force, sensing elements can be placed on a glove, directly onto a hand, or onto a handle or grip surface.

Although there are clear benefits to using thinfilm force sensors for certain applications, sensor performance and reliability have been uncertain. In order to study the capabilities of various sensor types, researchers have conducted a variety of evaluation tests [8-17]. No single study, however, has provided a comprehensive analysis of performance under both static and dynamic loading, combined with information about sensor performance in realistic test environments. The purpose of the current paper is to examine three different thin-film force sensors under both controlled laboratory conditions and in a real gripping situation to give an indication of each sensor's static accuracy, hysteresis, repeatability and drift errors, dynamic accuracy and drift errors, and the effects of shear loads and surface curvature. Equations used to calculate errors are all given in Appendix 2. Additionally, comments will be made on the advantages and disadvantages found when using each sensor type for a genuine grip force measurement. 


\subsection{Sensor types}

Two of the sensors tested in this study were manufactured by Tekscan Inc. These force sensors utilize a semi-conductive ink that is applied between electrical contacts and thin polyester sheets, creating a sensor with a resultant thickness of $0.1 \mathrm{~mm}$. These sensors respond to a change in force with an approximately linear change in resistance. The sensors are available in a large number of configurations, and for this study a matrix arrangement (F-Scan 9811) with a pressure rating of $0-517 \mathrm{kPa}(75 \mathrm{psi})$ and a single load cell (Flexiforce) with a force rating from $0-111 \mathrm{~N}$ (25lbf) were used. Each 9811 and Flexiforce sensing element has a sensitive region with an area of approximately $50^{2}$ and $71 \mathrm{~mm}^{2}$, respectively, but the 9811 software automatically assumes equal pressure in the non-sensitive regions surrounding the active area of the sensing elements. The 9811 sensor has a 6 by 16 matrix of sensing elements, resulting in 96 simultaneous force measurements across the sensor, and the sensor can be cut between its six columns to help aid its placement. The 9811 sensor comes as a complete hardware and software package, including a sensor connection box weighing $153 \mathrm{~g}$ to which the sensor must be attached. Flexiforce sensors are sold without any hardware or software, but only a simple circuit with power supply and a means of measuring the output voltage are required (Fig. 1). The 9811 and Flexiforce sensors are displayed to the same scale in Figs 2(a) and (b), respectively.

The third sensor considered was created from a pressure-sensitive material produced by Peratech Ltd. This material is known as a quantum tunnelling composite (QTC), and changes from a near perfect insulator to a conductor when deformed. Compression, twisting, or stretching of the QTC will change its conductivity, resulting in a material that can be used for force measurement. QTC is available as a $1-\mathrm{mm}$ thick rubbery material called the Pill Substrate and as an extremely thin paper-like substance referred to as Next Generation QTC. The latter was used to make individual sensors for this study. For QTC, electrodes and circuitry must be created by the user, offering complete control over sensor size and shape.

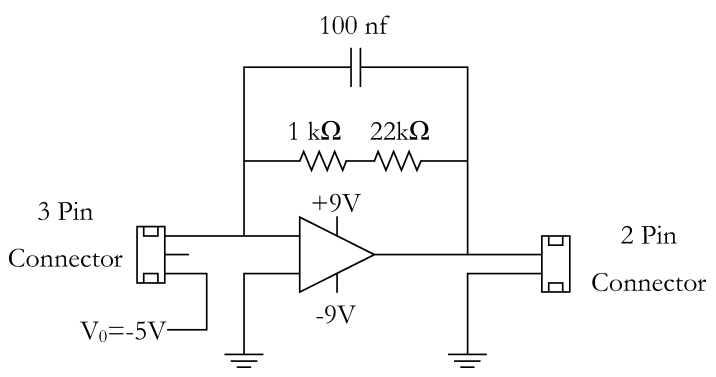

Fig. 1 Circuit used with Flexiforce sensors

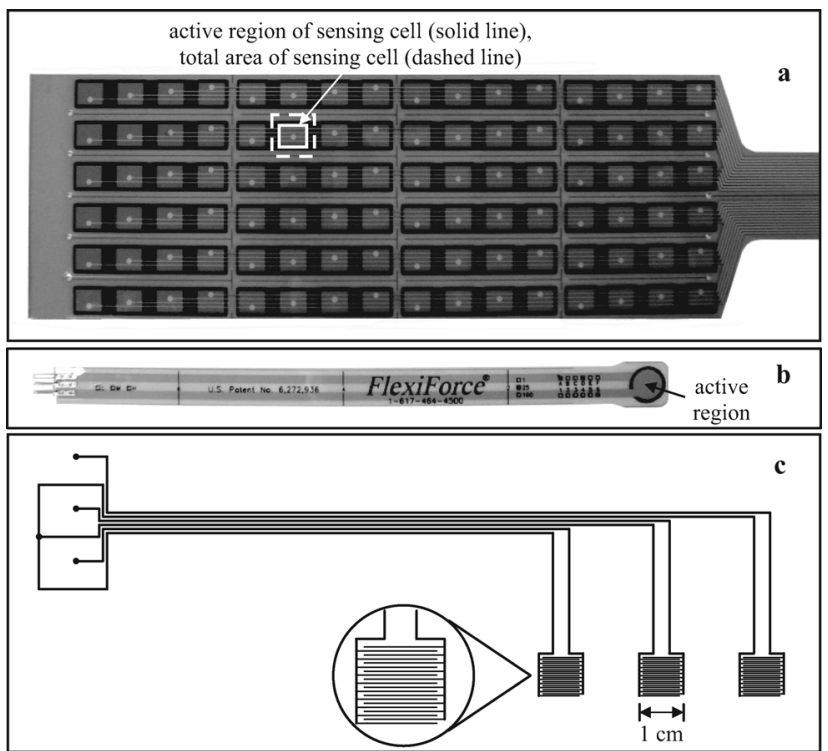

Fig. 2 (a) F-Scan 9811, (b) Flexiforce sensors, and (c) example schematic for a QTC electrode for three finger sensors

Circuitry is simple, consisting of a power source, resistor, and an electrode with QTC material connected in series. Voltage changes caused by sensor deformation were measured across the resistor. The electrodes were made by etching copper ( $17.5 \mu \mathrm{m}$ thickness) onto an insulated polyester material. A sample electrode is shown in Fig. 2(c). To make the sensor, QTC material was placed over the square interlocking region of the electrode (the enlarged portion of Fig. 2(c)). All of the QTC sensors used in the performance studies had square electrodes $(10 \times 10 \mathrm{~mm})$.

\section{SENSOR EVALUATION}

\subsection{Calibration}

Prior to the evaluation tests, all sensors were calibrated. Manufacturer's guidelines were used where appropriate and alternative calibration methods were developed when necessary. The 9811 sensor was equilibrated prior to calibration [18]. For equilibration, a custom-made bladder device was pressurized against the sensor to apply a uniform pressure of $310 \mathrm{kPa}$. The output from the 96 sensing elements was recorded and cell sensitivity variations were compensated by individual scale factors in the 9811 software. For calibration, the bladder device was again used to apply the same uniform load and the Tekscan software matched sensor output to the applied load. The equilibration and calibration loads were chosen as approximately 60 per cent of the maximum expected during the real grip scenario of interest (a golf shot), as suggested by the manufacturer. During the course of testing the 
sensor, it was determined that sensor drift during the calibration procedure introduced a systematic error to the measurements. A correction for this error is introduced later in section 2.2.2.

The Flexiforce and QTC sensors were calibrated by establishing the relationship between applied load and sensor output. Eleven weights ranging from 0.37 to $11.2 \mathrm{~kg}$ were applied to each of the sensors via a stainless steel cylindrical applicator (diameter $10 \mathrm{~mm}$, sized to fit within the active area of the sensors) with a thin $(2 \mathrm{~mm})$ rubber layer on the surface in contact with the sensor. The compliant layer was inserted to ensure that the load was evenly distributed across the active sensing area, avoiding local high pressures at edges, for example. Prior testing had shown that thin film sensors do not react well to large loads applied through sharp edges. Sensor output was measured after each change of mass during four load-unload cycles. A linear curve fit passing through zero was applied to the Flexiforce data and a second order or higher polynomial curve fit passing through zero was applied to the QTC data. Example calibration curves are shown in Figs 3(a) and (b), with r-squared and standard error of estimates for the Flexiforce curve of 0.94 and 0.41 , respectively, and for QTC 0.95 and 0.76 , respectively. Slight nonlinearities were noted near the Flexiforce load limit, but the effect of this was limited by including extra load points below 40 N. A cubic curvefit is shown in Fig. 3(a) for comparison, indicated by a dashed line. Overall, the linear fit suggested by the manufacturer is quite reasonable, especially for the lower force values expected in the golf tests. Additionally, the variations seen in sensor output at each load are mainly due to hysteresis effects which will be discussed later. As for the 9811 sensor, it was again found that sensor drift during the calibration procedure introduced systematic errors for which a correction is made.

\subsection{Methods and results}

For the accuracy, hysteresis, drift, and repeatability tests, three sensing elements on each of three 9811 sensors were used ( 9 sensing elements tested), and four of each of the Flexiforce and QTC sensors were examined. For the shear and curved surface tests, three sensing elements of each sensor type were studied.
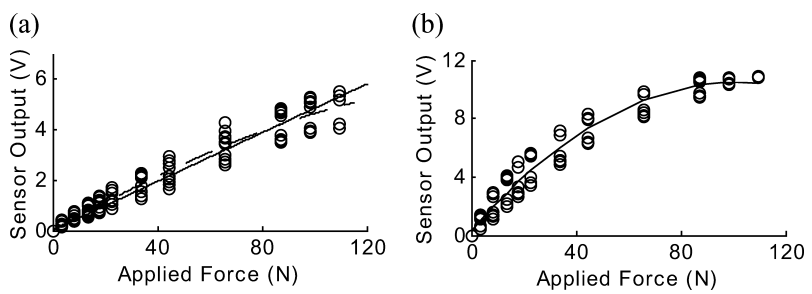

Fig. 3 Example calibration curves from (a) Flexiforce and (b) QTC sensors

\subsubsection{Quasi-static accuracy and hysteresis tests}

Accuracy and hysteresis errors were found by using a Lloyd tensile-compression testing machine to apply compressive loads to the sensors. During each test, loads were applied at rate of $1 \mathrm{~mm} / \mathrm{min}(\sim 1 \mathrm{~N} / \mathrm{s})$ and a load cell on the test machine (accuracy error $<1$ per cent) sampled the applied load at $10 \mathrm{~Hz}$. For the 9811 sensor, loads from 0 to $21 \mathrm{~N}$ were applied via a square applicator $(4.8 \times 4.8 \mathrm{~mm})$ that was smaller than the active area of the sensing element. The sensor was sampled at $120 \mathrm{~Hz}$ with ten repeat tests run on each sensing element.

During each test of the Flexiforce and QTC sensors, loads from 0 to $105 \mathrm{~N}$ were applied via the same cylindrical applicator used for calibration. The larger load values were chosen because of the higher load ratings and calibration levels for these two sensors compared with the 9811. The sensor output was recorded by a digital oscilloscope at $25 \mathrm{~Hz}$. The test was repeated on each sensor ten times. The force sensor data were then resampled to $10 \mathrm{~Hz}$ to match the load cell output, and each sensor's output was aligned with the applied load. Accuracy of the sensor output was then calculated as the difference between applied and measured forces divided by applied load as in equation (1) (Appendix 2).

Hysteresis can be defined as the maximum difference in sensor output for a given force level during loading and unloading [5, 19]. The hysteresis error was determined by using the data from the same quasi-static tests. Third-order polynomials were fitted to each load-unload curve (applied load versus sensor output) and hysteresis error was computed using equation (2).

The quasi-static tests showed that for identical loads, sensor output was higher during unloading than loading. For Tekscan 9811 and QTC, the sensors underestimated the applied load for the entire load-unload cycle, but to a lesser extent while unloading. These systematic errors indicate a problem with the calibration and this will be addressed shortly in section 2.2.2. Figure 4 shows data from the quasi-static loading and unloading of a 9811 sensor in two formats suitable for quantification of accuracy and hysteresis errors. Figure 4(a) shows uncorrected sensor output, and Figs 4(b) and (c) were corrected for systematic errors caused by sensor drift during calibration.

\subsubsection{Static repeatability and drift tests}

Static tests were also conducted to determine sensor repeatability and drift errors. For these tests, loads equivalent to approximately $300 \mathrm{kPa}$ were applied. During the 9811 repeatability tests, $5 \mathrm{~s}$ of data were collected at $264 \mathrm{~Hz}$ (the system maximum sampling rate) and the test was repeated ten times. The Flexiforce and QTC tests were conducted with sensor output being 

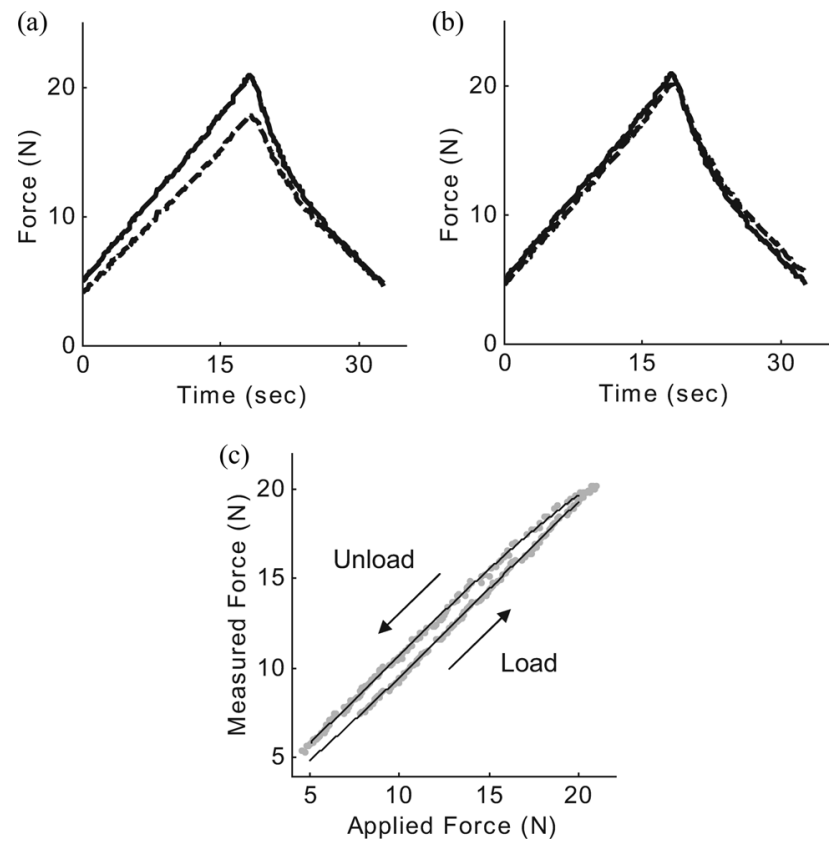

Fig. 4 Quasi-static loading and unloading of a 9811 sensor, (a) uncorrected data, (b) sensor output corrected for calibration drift error, - applied force, - - - measured force versus time, and (c) measured versus applied force for hysteresis calculation, - measured points, - curve fit

acquired through a standard signal analyser at $1280 \mathrm{~Hz}$ for five seconds. Twenty repeat tests were conducted.

The 'Starting Point' (the point where the load had been fully applied to the sensor) was determined based on changes in the slope of the force curve. The repeatability of each sensor output was compared using the output at $0.5 \mathrm{~s}$ after this point according to equation (3), in which the difference between the sensor output for a given run and the mean output for all runs with that particular sensing element is normalized by the mean output.

The drift tests conducted were similar to the repeatability tests, but with sensor output being measured for $65 \mathrm{~s}$. Ten repeats were carried out for each sensor, with the 9811 sensor sampled at $264 \mathrm{~Hz}$, and Flexiforce and QTC at $640 \mathrm{~Hz}$. Drift errors at $t=1,10$, and $60 \mathrm{~s}$ after the Starting Point were calculated using equation (4), where the difference between the sensor output at time $t$ and the Starting Point was normalized by the sensor output at the Starting Point. Example drift test data for each sensor are displayed in Fig. 5, with the force ratio indicating the ratio between the measured load and the measured force output at the Starting Point.

When drift errors were calculated, it was found that the Flexiforce sensor output stabilized almost instantly after a static load was applied, whereas the outputs from both the Tekscan 9811 and, in particular, QTC
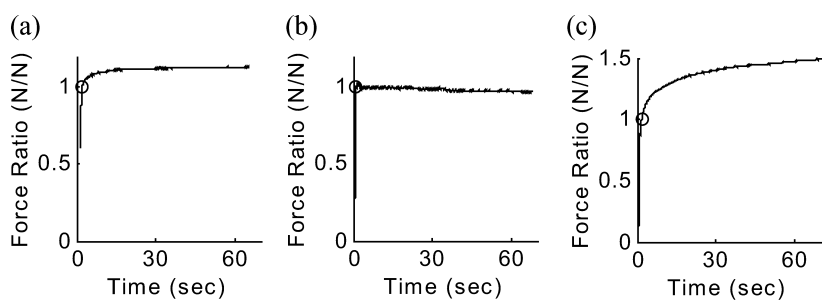

Fig. 5 Example drift test results from (a) 9811, (b) Flexiforce, and (c) QTC sensors; $\bigcirc$ indicates the 'Starting Point' from where drift is calculated

sensors were still increasing $60 \mathrm{~s}$ after the load had been applied.

The large drift error associated with the Tekscan 9811 sensor is problematic because, during the equilibration and calibration process, a uniform load is applied to the sensor for approximately $45 \mathrm{~s}$ before the calibration point is determined. The sensor drift during calibration produces a systematic error in sensor output, causing the sensor to underestimate the value of applied force. In order to account for this problem, a correction factor was found by minimizing the area between the applied and measured load curves during the quasi-static test. It was determined that a scale factor of 1.13 was optimal for minimizing the area. Additionally, although the exact time needed for calibration varies, the sensor drift error at $45 \mathrm{~s}$ was determined to be nearly 15 per cent, which is very close to the correction factor chosen. A similar correction was made for both the Flexiforce and QTC sensors as well, with scale factors of 0.956 and 1.14 being used, respectively. In all of the data presented in the current paper for each of the three sensors (including figures), these correction factors were used to adjust the sensitivity obtained from the manufacturer's recommended method of calibration.

Furthermore, during the repeatability and drift tests, it was noted that both Flexiforce and QTC sensors tended to underestimate the value of the applied load. This was most likely due to the fact that the sensors are calibrated by matching sensor output to applied loads during a full load-unload cycle. Consequently, loadings that include no hysteresis effects will be underestimated. If the sensors are to be used for measurements of monotonically increasing or decreasing loads, it is recommended that an alternative calibration method be used, with data points collected for increasing or decreasing loads only.

\subsubsection{Curved surface tests}

Thin-film force sensors are often required to bend around curved surfaces so it is important to understand the effect of such deformation on output. Four different surfaces were selected for this test - flat and with diameters of curvature of 30,25 , and $20 \mathrm{~mm}$. 
Seven loads were applied via the same applicators used in previously described tests to three sensors of each type. For the Tekscan 9811 sensor tests, loads varied between 0 and $33 \mathrm{~N}$, and for the Flexiforce and QTC tests the loads ranged from 0 to $110 \mathrm{~N}$. Three repeats of each test were performed.

In each test run, sensor output at zero load was recorded in order to measure preloads caused by sensor deformation. In all cases, the apparent preload measured on the curved surfaces was under $1.5 \mathrm{~N}$. For each test run, the sensor sensitivity was calculated from a plot of voltage output versus applied load (following the subtraction of the preload) using a linear curve-fit. The difference in sensitivity between the flat and curved surfaces was computed for each diameter of curvature and normalized by the sensitivity measured from the flat surface, as in equation (5), and is listed as surface curvature error. Surface curvature did not appear to have a huge effect on Tekscan 9811 or Flexiforce force measurements, but produced a rather large decrease in sensor output for the QTC sensors.

\subsubsection{Comparison with data from literature}

A summary of sensor characteristics under static loading conditions is given in Table 1. It should be noted that similar data from some of the sensor evaluation tests have been published previously [20], but some refinement has since been made in a number of the

Table 1 Results of static evaluation tests; values shown as percentages, with standard deviation in parentheses

\begin{tabular}{lccr}
\hline Error & Tekscan 9811 & Flexiforce & \multicolumn{1}{c}{ QTC } \\
\hline Accuracy & $6.7(4.8)$ & $10(3.5)$ & $13(2.8)$ \\
Hysteresis & $6.0(1.5)$ & $6.3(2.9)$ & $20(3.2)$ \\
Repeatability & $3.3(0.73)$ & $4.5(0.57)$ & $7.1(2.2)$ \\
Drift $(1 \mathrm{~s})$ & $+4.1(2.8)$ & $+1.6(2.1)$ & $+10(3.3)$ \\
Drift $(10 \mathrm{~s})$ & $+11(4.7)$ & $+1.5(2.6)$ & $+34(6.8)$ \\
Drift $(60 \mathrm{~s})$ & $+15(6.4)$ & $-0.51(3.2)$ & $+59(11)$ \\
Surface curvature & $-1.4(3.8)$ & $+2.1(2.0)$ & $-11(8.0)$ \\
$\quad(30 \mathrm{~mm})$ & & & \\
Surface curvature & $-5.2(1.9)$ & $+0.95(2.7)$ & $-25(6.5)$ \\
$\quad(25 \mathrm{~mm})$ & & & \\
$\quad \begin{array}{l}\text { Surface curvature } \\
\quad(20 \mathrm{~mm})\end{array}$ & $-1.0(3.4)$ & $+2.9(4.0)$ & $-31(8.0)$ \\
\hline
\end{tabular}

error calculations. Table 2 is a summary of results from similar studies that looked at various Tekscan matrix and Flexiforce sensors. The authors are unaware of any previous published studies of the QTC material. Although the data in the table originate from several different types of Tekscan matrix sensors, in most cases they behaved in a very similar manner to the 9811 matrix sensors in this study. There were two cases in which rather high hysteresis values were found, but for those studies hysteresis was either calculated as the maximum value found in all test runs for a given sensor [13], or only a single sensor was considered in the study [15].

Only one paper was found that considered Flexiforce sensor performance, and in that case it was for the low force (0-1 lbf) variety [12]. Even so, hysteresis and repeatability were very similar, but the drift errors are difficult to compare based on how they were presented. The current paper also reported the effects of surface curvature on sensor output for radii of curvature from 8.0 to $51.7 \mathrm{~mm}$ and found that preload increased with decreasing radius, while the sensitivity of the sensor decreased for radii below $32.5 \mathrm{~mm}$. It should be noted, however, that as this is a lowforce sensor these variations in sensor output may have been negligible for the $25 \mathrm{lbf}$ sensors used in the current study.

\subsubsection{Shear test}

A novel static load test was conducted on the sensors to determine the effect of shear load on sensor output. No literature has been found by the authors indicating how thin-film sensors react to this type of loading. Shear forces are often present in dynamic gripping actions, so it is important to ascertain how these sensors behave under this loading condition. The shear loads chosen for this test were based on peak shear forces of around $500 \mathrm{~N}$, which occur between the hands and the grip during golf shots [21, 22], and then scaled based on the area over which they are applied. A three-legged circular load carrier (cylindrical legs, $6 \mathrm{~mm}$ diameter, $120^{\circ}$ spacing with $2 \mathrm{~mm}$-thick rubber layer on each leg) was used to apply normal loads of 9.2 and $15.75 \mathrm{~N}$ to each sensor, as shown in Fig. 6(a).

Table 2 Results from static evaluation tests in literature for comparison

\begin{tabular}{|c|c|c|c|c|}
\hline & Accuracy & Hysteresis & Repeatability & Drift \\
\hline \multirow{3}{*}{$\begin{array}{l}\text { Tekscan matrix- } \\
\text { type sensor }\end{array}$} & $2-3 \%[8]$ & $42 \%[13]$ & As low as $2 \%[\mathbf{1 6}]$ & $12 \%$ for $5-20 \min [13]$ \\
\hline & $6.5 \%[10]$ & $21 \%[\mathbf{1 5}]$ & $2.3 \%[\mathbf{1 7}]$ & $\begin{array}{l}12 \% \text { at } 1 \mathrm{~min}, 19 \% \text { at } 15 \mathrm{~min} \\
{[\mathbf{1 5}]}\end{array}$ \\
\hline & $\begin{array}{l}1.3-5.8 \%[11] \\
8.5 \%[13]\end{array}$ & $5.6-8.5 \%[17]$ & & $39 \%$ for $2 \mathrm{~h}[\mathbf{1 6}]$ \\
\hline $\begin{array}{l}\text { Flexiforce sensor } \\
\quad(1 \mathrm{lbf})\end{array}$ & - & $5.4 \%[12]$ & $2.3-6.6 \%[12]$ & $\begin{array}{l}\text { Sensor reaches } 96 \text { and } 98 \% \text { of } \\
\text { stable level at } 5 \text { and } 10 \mathrm{~min} \text {, } \\
\text { respectively [12] }\end{array}$ \\
\hline
\end{tabular}


(a)

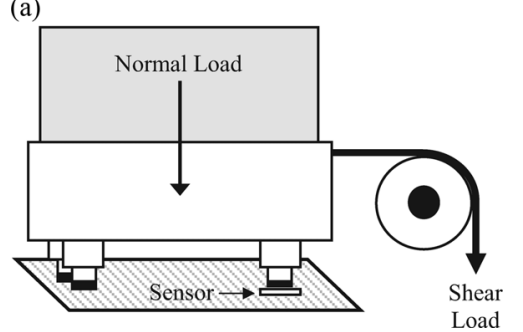

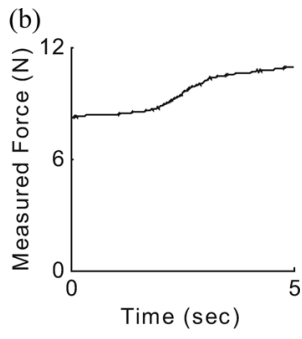

Fig. 6 (a) Shear force test set-up, and (b) example QTC shear force test with $9.2 \mathrm{~N}$ normal and $3.2 \mathrm{~N}$ shear loads applied to the sensor

Data were collected from each sensor for $5 \mathrm{~s}$ and, after the data collection had begun, a shear load of 1.6 or $3.2 \mathrm{~N}$ was applied to each sensor via the load carrier (example sensor output for a QTC shear test is shown in Fig. 6(b). To determine the change in output due to shear over each $5 \mathrm{~s}$ test, the overall change in sensor output was first calculated by subtracting the mean sensor output from the last $0.5 \mathrm{~s}$ of data from the mean for the first $0.5 \mathrm{~s}$. The change in output due to drift was then estimated and this was subtracted from the overall change in sensor output to give the contribution due to the application of a shear force. The shear sensitivity was then determined according to equation (6) by dividing the contribution to the sensor output due to shear by the applied shear force.

Table 3 displays the results from the shear force test. Note that values are not given for shear sensitivity of QTC for the higher normal loads. This was due to the sensor overloading during the majority of tests with the higher normal force as soon as the shear load was applied. All three sensors show considerable sensitivity to shear (increased sensor output), although the effects of the magnitudes of shear and normal forces differ for each. The Tekscan 9811 sensor shear sensitivity increases with shear magnitude but appears independent of normal force, the Flexiforce sensor shear sensitivity appears independent of shear and normal load, and the QTC sensor shear sensitivity was nearly constant for changes in shear load for the lower normal force. At this point, the underlying causes for these variations in sensor behaviour with shear and normal loading are not clear.
An additional problem with the QTC sensor was discovered during the evaluation tests. As each QTC sensor calibration or test proceeded, the sensor sensitivity decreased. This decrease in sensitivity affected the sensor's accuracy over time. It is believed that the decrease in sensitivity contributed to the high accuracy and curved surface errors that were measured for this sensor.

\subsubsection{Dynamic accuracy and drift tests}

A novel set of tests were designed to examine the response of the sensors under a dynamic load. The authors have not found similar tests in literature. Sensors were placed under one of the three legs of a load carrier (described in section 2.2.5) which rested on top of a platform, as shown in Fig. 7. The platform was fixed to an electromagnetic shaker for vertical excitation, with stability provided by restricting the motion of two of the legs (Fig. 7(b)). With this set-up, onethird of the total static and dynamic load applied to the carrier was directed through the test sensor via the $6-\mathrm{mm}$ diameter cylindrical leg. Sinusoidal loads were applied to the sensor at frequencies of 15,60 ,
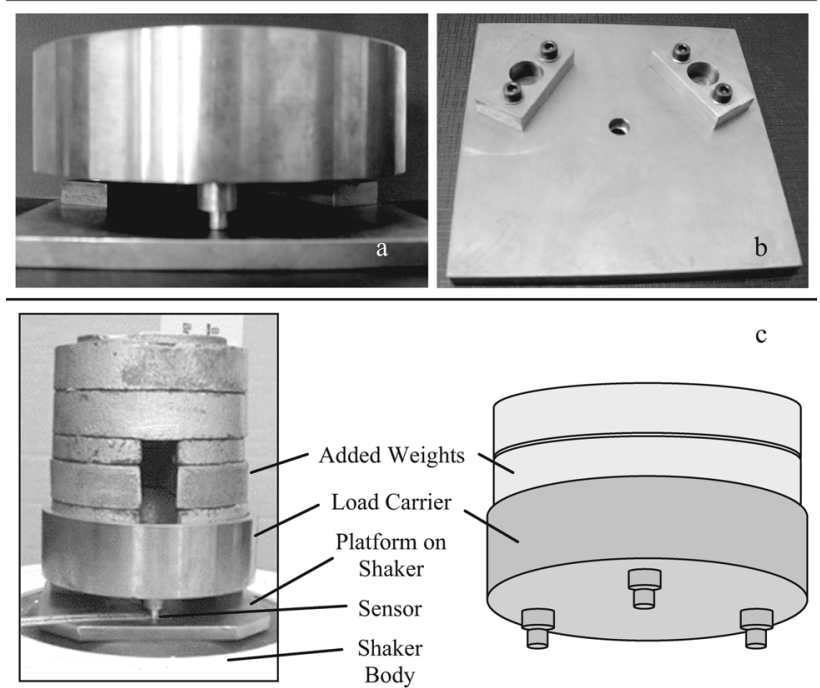

Fig. 7 (a) Load carrier, (b) platform, and (c) entire set-up used for dynamic test

Table 3 Results of shear force tests; values shown are percentage change in force output due to shear per unit applied shear load, and standard deviation is listed in parentheses

\begin{tabular}{lllllll}
\hline & \multicolumn{2}{c}{ Tekscan 9811 } & \multicolumn{2}{c}{ Flexiforce } & \multicolumn{2}{c}{ QTC } \\
\cline { 2 - 7 } & Shear & Shear & Shear & Shear & Shear & Shear \\
Load & $1.6 \mathrm{~N}$ & $3.2 \mathrm{~N}$ & $1.6 \mathrm{~N}$ & $3.2 \mathrm{~N}$ & $1.6 \mathrm{~N}$ & $3.2 \mathrm{~N}$ \\
\hline Normal 9.2 N & $+28(19)$ & $+49(19)$ & $+37(22)$ & $+33(20)$ & $+25(13)$ & $+30(12)$ \\
Normal 15.8 & $+24(18)$ & $+51(19)$ & $+41(26)$ & $+37(21)$ & - & - \\
\hline
\end{tabular}


and $100 \mathrm{~Hz}$, such that the $6.3 \mathrm{~kg}$ load achieved nominal maximum peak accelerations of 2.4, 4.8, and $7.1 \mathrm{~m} / \mathrm{s}^{2}$ measured by an accelerometer (accuracy error of $1-2$ per cent over the frequency range used) placed on the load carrier just above the pressure sensor. The load applied through the sensor follows readily from the product of the mass and the accelerometer measurement. Each dynamic load and frequency combination was repeated five times on each sensing element tested.

The dynamic loads applied to the 9811 sensor were sampled at $264 \mathrm{~Hz}$. These loads were applied for a total of $10 \mathrm{~s}$, but $20 \mathrm{~s}$ of data were recorded to investigate sensor output drift during the test. For the Flexiforce and QTC sensors, a signal analyser was used to collect the output from the sensors and accelerometers at a sample rate of $1.28 \mathrm{kHz}$. The dynamic load was applied for a total of $5 \mathrm{~s}$, but 10-15 s of data were recorded. An example from the Flexiforce dynamic test is displayed in Fig. 8.

Dynamic drift error was calculated using equation (7) and indicates the change in sensor output over a 10-s interval during the test. The initial force was found by averaging the first $0.25 \mathrm{~s}$ of the sensor output and the final force was the mean of the last $0.25 \mathrm{~s}$ of the 10$s$ interval. Dynamic drift error was then evaluated as the change in mean force from the beginning to end of the 10-s interval divided by the initial force. Mean

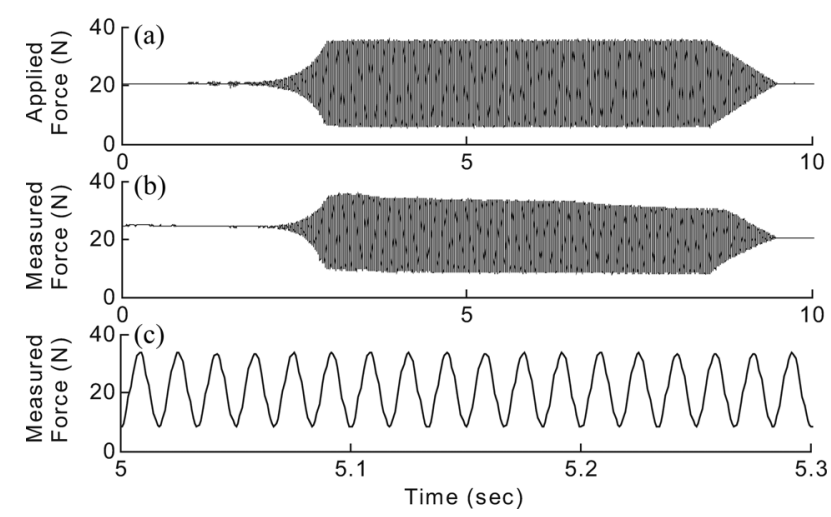

Fig. 8 Example Flexiforce dynamic test $(\sim 15 \mathrm{~N}$ at $60 \mathrm{~Hz})$; (a) static load plus dynamic load measured by accelerometer, (b) Flexiforce sensor output, and (c) expanded section of trace (b) dynamic drift error was found to be between -2.9 to +13 per cent for the three sensor types and large standard deviations indicate that this was quite variable from test to test.

An 8-s segment of each 9811 load curve and a 4-s segment of each Flexiforce and QTC load curve were found for which the peak oscillating load was applied the entire time. The static load and low frequency drift were filtered out of each segment using MATLAB fast Fourier transform (FFT) and inverse FFT (IFFT) functions. The RMS force measured from each sensor was compared with the applied RMS force calculated from the accelerometer output for each test. The dynamic accuracy of the sensors was then calculated as the difference between measured and applied loads normalized by applied load as in equation (8).

A summary of all dynamic test data is shown in Table 4. For dynamic loads, all sensors exhibited a much larger variation in output between runs as compared with the static tests. Large standard deviations were computed for the dynamic accuracy errors for all three sensors, with QTC having the largest. It was found that sensor output decreased with increasing frequency of applied load for the Flexiforce and QTC sensors, while the 9811 sensor underestimated the applied load to a similar magnitude for all three load frequencies $(15,60$, and $100 \mathrm{~Hz})$. It was also noted that the run order affected the dynamic accuracy of the sensors. The calculated dynamic accuracy of QTC sensors decreased as the testing progressed, while for Flexiforce sensors it increased. In the case of the 9811 sensor, different sensors seemed to show different run order effects. Decreasing output with continued testing may indicate sensor degradation, while increasing output with continued testing is harder to explain but may represent some residual drift-like effects.

Sensor performance during the dynamic accuracy tests may have been improved with the addition of a compliant layer between the sensor and the rigid load applicator (leg of the load carrier) and this would be recommended for future tests. Additionally, change in sensor sensitivity due to repeated use has most certainly played a role in the measured mean and standard deviation of dynamic accuracy.

Table 4 Results of dynamic evaluation tests; values shown as percentages, with standard deviation in parentheses

\begin{tabular}{llllll}
\hline & \multicolumn{2}{c}{ Dynamic Drift $(10 \mathrm{~s})$} & Accuracy & \multicolumn{3}{c}{ Dynamic accuracy } \\
\cline { 2 - 6 } & Based on all frequencies & $0 \mathrm{~Hz}$ & $15 \mathrm{~Hz}$ & $60 \mathrm{~Hz}$ & $100 \mathrm{~Hz}$ \\
\hline Tekscan 9811 & $+13(5.9)$ & $6.7(4.8)$ & $-57(9.7)$ & $-64(10)$ & $-58(11)$ \\
Flexiforce & $-10(5.0)$ & $10(3.5)$ & $-16(21)$ & $-25(14)$ & $-25(18)$ \\
QTC & $-2.9(15)$ & $13(2.8)$ & $+28(62)$ & $-15(40)$ & $-38(34)$ \\
\hline
\end{tabular}




\section{APPLICATION - MEASURING GRIP FORCE IN GOLF}

To evaluate aspects of sensor performance in practical applications, the three sensors were also used to measure grip force during a golf shot. A typical driver shot was chosen as it is a fairly repeatable event but also capable of testing sensor limitations. The tapered grip of a standard golf club can make sensor attachment difficult as the sensor is required to bend to conform to the curvature of the golf grip, the sensor has to withstand high shear loads due to large centrifugal forces created during the shot, applied force can change rapidly over a short period of time, and peak forces vary depending on sensor location and golfer.

Due to variations in sensor size and shape, each of the three sensor types were used in different ways but with the same goal - to measure grip force during a golf shot. The following sections provide a description of how each sensor type was utilized, some sample data, and comments on sensor performance based on player tests.

\subsection{Sensor configuration for player tests}

\subsubsection{Tekscan 9811}

The Tekscan 9811, with its rectangular matrix of 16 by 6 sensing elements, was attached directly to the golf grip. To facilitate this task, the sensor was cut along its columns, leaving 6 strips of sensing elements that were connected at one end, which could then be evenly spaced running down the length of the golf club grip. Double-sided tape was used to attach the sensor to the grip and thin strips of electrical tape were positioned over the non-sensitive areas of the sensor to hold it in place. Micropore tape (a permeable, nonwoven, surgical, synthetic adhesive manufactured by $3 \mathrm{M}$ ) was wrapped around the sensor to help protect it from potential damage by shear forces produced during a golf shot. When attached to the golf grip, 84 of the 96 sensing elements rested entirely against the grip surface and it is those elements that were considered in the analysis. Images of the sensor on the grip are displayed in Figs 9(a) and (b). This configuration was good for total force measurements but made it difficult to associate data with particular locations on the hand.

\subsubsection{Flexiforce}

Being a single load cell type sensor, the Flexiforce sensor has the benefit that any number of sensors can be used simultaneously and be positioned independently of one another. However, as the number of sensors increases, the complexity of the data acquisition system and the time spent calibrating sensors increase.
For measuring grip force, a 32-channel data acquisition system was employed and code to control it was written in Visual Basic. Similar configurations have been used previously to measure grip force $[4,23,24]$. All 32 channels were utilized during the golf tests, with one channel reserved to record sound data (in order to determine the moment of impact), and all other channels connected to force sensors. The 31 -force sensors were then attached to strategic locations on two golf gloves as shown in Figs 9(c) and (d). Double-sided and micropore tape were again used to help position, secure and protect the sensitive area of each sensor. The remainder of each sensor was directed along the most convenient route to the back of the hand using hand-sewn loops of elastic thread as a guide. This arrangement was good for associating grip force with particular locations on the hand, but not as good at total force measurements as the 9811 sensors because a smaller portion of the hand-grip interface is covered by the sensors.

\subsubsection{Peratech QTC}

Next generation QTC is supplied as a sheet of force sensitive material from which the user creates sensors of any desired shape and size. Like the Flexiforce sensors, if the user wants independent measurements to be taken at multiple locations, a data acquisition system and associated software are necessary. This configuration has the advantage of being able to monitor force at individual regions of the hand, with the added benefit of being able to determine sensor size and shape.

The unlimited layout options available for this sensor meant that sensors could be created in order to be attached to either the grip or gloves. For the player tests, the 32-channel system was used to control 31 sensors attached to two golf gloves as shown in Figs 9(e) and (f). All of the 27 sensors on the fingers were square in shape $(10 \times 10 \mathrm{~mm})$, and the sensitive area of the four sensors positioned on the palms were enlarged to cover a greater area. The shape of each sensor was designed specifically for this application, attempting to minimize interference of the sensor with the golfer's grip of the club and to enhance ease of attachment to the gloves.

\subsection{Player tests}

Golfers of varying ability (handicaps from 0 to 22 plus two players new to the game and without handicap) participated in the grip force tests. Each golfer hit 10-12 shots with a standard driver (graphite shaft, titanium head, $9.5^{\circ}$ loft) in an indoor netted enclosure off an artificial turf matt with a rubber tee. In all cases, the golfer was required to wear two golf gloves (cabretta palm and synthetic leather top), and all wires or cables associated with the sensors were led up the 

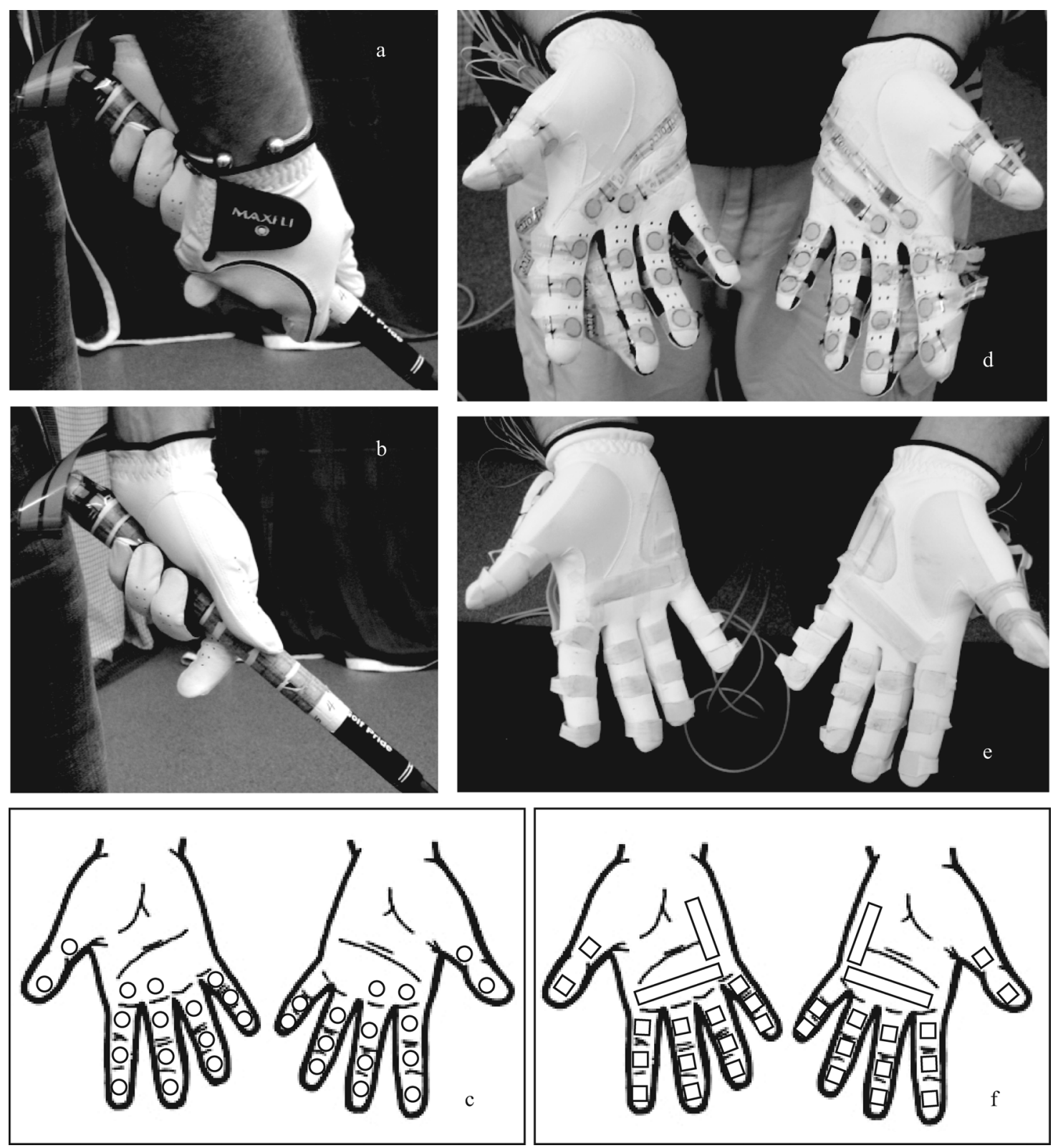

Fig. 9 Sensor application for golf grip force measurement - (a) and (b) Tekscan 9811 sensor on the grip, (c) and (d) Flexiforce, and (e) and (f) QTC sensors on gloves with sensor location indicated

golfers' arms and over their shoulders. With sufficient time given to the golfers to adjust to the placement of the cables and the feel of the sensors, they reported that the sensors did not interfere with their customary swing.

Acquisition of data from the sound level meter and the force sensors was triggered simultaneously prior to each shot. The microphone was located as close as possible to the tee and the sound trace was used to determine the moment of impact in the force data. For tests using the 9811 sensor, the force data from the 84 sensing elements on the grip were summed, and example total grip force curves for four golfers from this test are shown in Fig. 10. In each plot, total force for all ten shots taken is aligned based on time of impact (noted as $0 \mathrm{~s}$ ). From the data, it is evident that each player, regardless of ability, has their own unique grip force 'signature', a very consistent and virtually unique grip force profile. This interesting phenomenon emerged from an early phase of the study and initial findings were reported previously [25].

Total force-time curves can be created for all three sensor types. The 9811 sensor covered a considerably larger area of the hand-grip interface, and so calculated total force values for this sensor were higher. By looking at normalized versions of the total force traces 

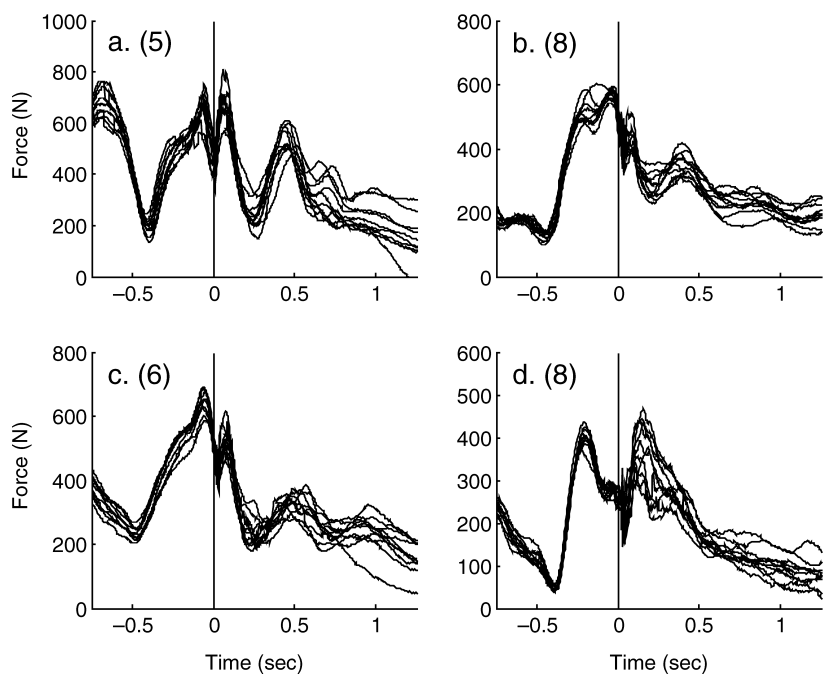

Fig. 10 Ten total grip force curves from four golfers measured with Tekscan 9811 sensors on golf club grip; player handicap is shown in parentheses and impact is shown as time $=0 \mathrm{~s}$

(normalization conducted by subtracting the mean and dividing by the standard deviation), the outputs of the three sensor types can be compared. Figure 11 is typical of the data obtained and shows the normalized mean total force for one golfer, measured by each sensor type. Previous tests conducted by Budney [22] and Budney and Bellow [26] indicated grip forces produced by both professional and amateur golfers at three regions of the hand. Summing the forces from those tests produces similar trends in the data, such as peaks occurring just before and after impact for the majority of golfers.

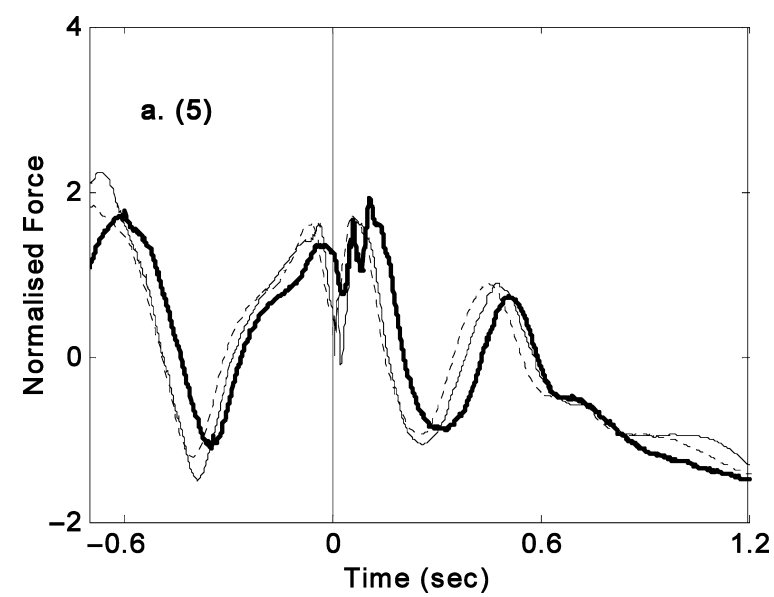

Fig. 11 Mean normalized total grip force for one golfer, output from each sensor type displayed and impact is shown as Time $=0 \mathrm{~s}$; --- 9811, Flexiforce, - QTC
As the grip force is a dynamic event, it is likely that the sensors underestimated the force due to the rapidly changing applied loads. Applied shear forces would have the opposite effect, causing the sensors to overestimate the force. Drift should not be a large factor due to the relatively short duration and changing locations of applied forces, and attempts were made to minimize the effect of bending the sensors around the grip by subtracting preloads (for 9811 sensors) from the sensor output. Notwithstanding the errors identified during the sensor evaluation tests and given the fact that several months elapsed between player tests for the different sensor types, it is notable that all three sensor types provided very similar and repeatable grip force signatures.

Sensor durability was also assessed based on the player tests. Each golfer took approximately 10-12 shots with the force sensors in place. The Tekscan 9811 and Flexiforce sensors typically lasted for 5-6 golfers before there was a large drop in sensor sensitivity and the QTC sensors typically lasted for three golfers. For the QTC sensors, it is difficult to be sure if it was the QTC material itself that was degrading, or if it was another part of the sensor such as the electrodes.

\section{CONCLUSIONS}

Three types of thin, flexible force sensors were subjected to a rigorous and comprehensive set of static and dynamic tests to determine their characteristics under varied loading conditions. In particular, novel tests were developed to evaluate shear force sensitivity and dynamic sensor behaviour. Each of these sensors was then adapted for use in a real, dynamic grip force measurement.

QTC sensors displayed quite large drift and curved surface errors. As a relatively undeveloped but still promising technology, the QTC sensors used in these tests did not perform as well as the commercial sensors tested. Tekscan 9811 and Flexiforce sensors had static errors that were not negligible but were acceptable given the advantages they offered over alternatives for the grip force application. Tekscan 9811 had a larger drift error than the Flexiforce sensor, and as a result the manufacturer's recommended calibration procedure had to be modified using the results from a series of quasi-static tests in order to produce an acceptable output. The Flexiforce sensor drift was minimal, and so such a problem did not exist. For dynamic loads, the sensitivities of both Tekscan 9811 and Flexiforce sensors were both measurably reduced compared with the sensitivity found using quasi-static loads, with some deviation also apparent, but again this limitation was outweighed by the advantages offered by 
these sensors over any alternatives for the grip force application.

The Tekscan 9811 and Flexiforce sensors proved to be durable during the player tests and the output for an individual golfer was very repeatable. As in the evaluation tests, the QTC sensors did not perform as well as the commercial sensors during real grip force measurements. One problem, apparent for all three sensors, was a loss of sensitivity with use and this requires some further investigation. Nevertheless, all three sensors provided useful force data, adding to the knowledge of what happens at the grip during a golf shot. In particular, the grip force signature phenomenon was revealed.

\section{ACKNOWLEDGEMENTS}

The authors would like to thank the golfers for their participation in the current study.

\section{REFERENCES}

1 Chadwick, E. K. J. and Nicol, A. C. A novel force transducer for the measurement of grip force. J. Biomech., 2001, 34(1), 125-128.

2 McGorry, R. W. A system for the measurement of grip forces and applied moments during hand tool use. Appl. Ergon., 2001, 32(3), 271-279.

3 Van der Kamp, M., Conway, B. A., and Nicol, A. C. A novel instrumented ring for the measurement of grip force adjustments during precision grip tasks. Proc. Instn Mech. Engrs, Part H: J. Engineering in Medicine, 2001, 215(H2), 421-427.

4 Yun, M. H., Kotani, K., and Ellis, D. Using force sensitive resistors to evaluate hand tool grip design. In Proceedings of the Human Factors Society 36th Annual Meeting, Santa Monica, CA, 1992, pp. 806-810.

5 Bray, A., Barbato, G., and Levi, R. Theory and practice of force measurement, 1990 (Academic Press, London).

6 Amis, A. A. Variation of finger forces in maximal isometric grasp tests on a range of cylinder diameters. J. Biomed. Eng., 1987, 9, 313-320.

7 An, K. N., Chao, E. Y. S., and Askew, L. J. Hand strength measurement instruments. Arch. Phys. Med. Rehabil., 1980, 61, 366-368.

8 Bachus, K. N., DeMarco, A. L., Judd, K. T., Horwitz, D. S., and Brodke, D. S. Measuring contact area, force, and pressure for bioengineering applications: using Fuji Film and TekScan systems. Med. Eng. Phys., 2006, 28(5), 483-488.

9 Wilson, D. C., Niosi, C. A., Zhu, Q. A., Oxland, T. R., and Wilson, D. R. Accuracy and repeatability of a new method for measuring facet loads in the lumbar spine. J. Biomech., 2006, 39(2), 348-353.

10 Wilson, D. R., Apreleva, M. V., Eichler, M. J., and Harrold, F. R. Accuracy and repeatability of a pressure measurement system in the patellofemoral joint. J. Biomech., 2003, 36, 1909-1915.
11 Hsiao, H., Guan, J., and Weatherly, M. Accuracy and precision of two in-shoe pressure measurement systems. Ergonomics, 2002, 45(8), 537-555.

12 Ferguson-Pell, M., Hagisawa, S., and Bain, D. Evaluation of a sensor for low interface pressure applications. Med. Eng. Phys., 2000, 22, 657-663.

13 Polliack, A. A., Sieh, R. C., Craig, D. D., Landsberger, S., McNeil, D. R., and Ayyappa, E. Scientific validation of two commercial pressure sensor systems for prosthetic socket fit. Prosthet. Orthot. Int., 2000, 24, 63-73.

14 Buis, A. W. P. and Convery, P. Calibration problems encountered while monitoring stumps/socket interface pressures with force sensing resistors: techniques adopted to minimise inaccuracies. Prosthet. Orthot. Int., 1997, 21, 179-182.

15 Woodburn, J. and Helliwell, P. S. Observations on the F-Scan in-shoe pressure measuring system. Clin. Biomech., 1996, 11(5), 301-304.

16 Werner, F. W., Green, J. K., Fortino, M. D., Mann, K. A., and Short, W. H. Evaluation of a dynamic intra-articular contact pressure sensing system. In 41st Annual Meeting, Orthopaedic Research Society, Orlando, Florida, 1995, p. 705.

17 Pavlovic, J. L., Takahashi, Y., Bechtold, J. E., Gustilo, R. B., and Kyle, R. F. Can the Tekscan sensor accurately measure dynamic pressures in the knee joint. In 17th Annual Meeting, American Society of Biomechanics, Iowa City, Iowa, 1993, pp. 135-136.

18 Tekscan. Tekscan user manual: sensing solutions for the 21st century, 2004, $309 \mathrm{p}$.

19 The Institute of Measurement and Control. Guide to the measurement of force. The Institute of Measurement and Control, 1998.

20 Schmidt, E. R., Roberts, J. R., and Rothberg, S. J. Thin, flexible sensors for grip force measurements in sport. In Asia Pacific Congress on Sports Technology, Tokyo, 2005, pp. 399-405.

21 Mather, J. S. B. The role of club response in the design of current golf clubs. In Proceedings of the 14th International Modal Analysis Conference, Michigan, 1996, pp. 397-403.

22 Budney, D. R. Measuring grip pressure during the golf swing. Res. Q., 1979, 50(2), pp. 272-277.

23 Nikonovas, A., Harrison, A. J. L., Hoult, S., and Sammut, D. The application of force-sensing resistor sensors for measuring forces developed by the human hand. Proc. Instn Mech. Engrs, Part H: J. Engineering in Medicine, 2004, 218, 121-125.

24 Kong, Y.-K. and Lowe, B. D. Optimal cylindrical handle diameter for grip force tasks. Int. J. Ind. Ergon., 2005, 35, 495-507.

25 Schmidt, E. R., Roberts, J., and Rothberg, S. Timeresolved measurements of grip force during a golf shot. In The engineering of sport 6 (Eds E.F. Moritz and S. Haake), 2006, pp. 57-62 (Springer, New York).

26 Budney, D. R. and Bellow, D. G. Evaluation of golf club control by grip pressure measurement. In Science of golf I, 1990, pp. 30-35 (E \& FN Spon, London).

27 BS PD 6461-3: 1995. General metrology: guide to the expression of uncertainty in measurement, 1995. 


\section{APPENDIX 1}

\section{Notation}

$F_{\text {ref }} \quad$ reference force measurement

$\tilde{F}_{\text {ref }} \quad$ filtered reference force measurement

$F_{\text {sens }} \quad$ sensor force measurement

$\tilde{F}_{\text {sens }}$

$F_{\text {shear }}$

$n$

$n_{1}$

$n_{\mathrm{r}}$

$n_{\mathrm{u}}$

$n_{0}$

$n_{1}$

$n_{1} \Delta t$

$n_{2}$

$N+1$

$S_{\mathrm{c}}$

$S_{\mathrm{f}}$

$T$

$\Delta F_{\mathrm{drift}}$

$\Delta t$

\section{APPENDIX 2}

The following equations were used to calculate the errors and sensitivities described, and are partially based on those indicated by Bray et al. [5] and The Institute of Measurement and Control [19]. In each test, $N+1$ samples are taken with a time increment $\Delta t$ giving a sample length $T$. Error terms are derived from force measurements, $F(n \Delta t)$. The subscripts ref and sens are used to indicate the reference measurement and the associated sensor output, respectively. The following equations indicate the error associated with measurements from each sensing element. The error values given in the text are typically quoted as the mean of the errors from all runs on all test sensing elements of each sensor type \pm the standard deviation [27].

$$
\text { Accuracy error: } \frac{1}{(N+1)} \sum_{n=0}^{N}\left[\frac{-\mid F_{\text {ref }}(n \Delta t)}{-F_{\text {sens }}(n \Delta t) \mid}\right] \times 100
$$

$$
\text { Hysteresis error: }\left[\begin{array}{c}
\max \left(\mid F_{\text {sens }}\left(n_{\mathrm{u}} \Delta t\right)\right. \\
\frac{\left.-F_{\text {sens }}\left(n_{1} \Delta t\right) \mid\right)}{\max \left(F_{\text {sens }}(0: T)\right)}
\end{array}\right] \times 100
$$

where $n_{\mathrm{u}}$ is the point in the unload half of the cycle when the applied load is the same as at $n_{1}$ in the load half of the cycle (i.e. $\left.F_{\text {ref }}\left(n_{\mathrm{u}} \Delta t\right)=F_{\text {ref }}\left(n_{1} \Delta t\right)\right)$. The maximum value is found from all possible matching pairs of $n_{\mathrm{u}}$ and $n_{\mathrm{l}}$ (facilitated by polynomial curve fit to experimental data).

$$
\text { Repeatability error: }\left[\frac{\mid \mathrm{F}_{\text {sens }}\left(n_{\mathrm{r}} \Delta t\right)}{-F_{\text {sens }}\left(n_{\mathrm{r}} \Delta t\right) \mid} \frac{\overline{F_{\text {sens }}\left(n_{\mathrm{r}} \Delta t\right)}}{}\right] \times 100
$$

where $n_{\mathrm{r}}$ is the single, chosen point after the Starting Point at which comparison is to be made. Repeated measurements on each test sensing element of each sensor enable the calculation of a mean value $\overline{F_{\text {sens }}\left(n_{\mathrm{r}} \Delta t\right)}$.

$$
\text { Drift error (static): }\left[\begin{array}{c}
F_{\text {sens }}(n \Delta t)- \\
\frac{F_{\text {sens }}\left(n_{0} \Delta t\right)}{F_{\text {sens }}\left(n_{0} \Delta t\right)}
\end{array}\right] \times 100
$$

where $n_{0}$ and $n$ indicate the points where the load is first applied and when the drift calculation is being made, respectively (i.e. $\left(n-n_{0}\right) \Delta t=1,10,60 \mathrm{~s}$ ).

$$
\text { Surface curvature error: }\left[\frac{S_{\mathrm{c}}-S_{\mathrm{f}}}{S_{\mathrm{f}}}\right] \times 100
$$

where $S$ is the sensor sensitivity calculated from a plot of voltage output versus known applied load (following the subtraction of the voltage output at zero load, i.e. due to bending preload), and subscripts ' $c$ ' and 'f' indicate test runs for the sensor on curved and flat surfaces, respectively.

\section{Shear sensitivity:}

$$
\left[\begin{array}{c}
1 / n_{1} \sum_{\substack{n=N-n_{1}+1 \\
n=n_{1}-1}}^{n=N} F_{\text {sens }}(n \Delta t) \\
-\left(1 / n_{1}\right) \sum_{n=0}^{n} F_{\text {sens }}(n \Delta t)-\Delta F_{\text {drift }} \\
F_{\text {shear }}
\end{array}\right] \times 100
$$

where $n_{1} \Delta t$ is a chosen length of time $(0.5 \mathrm{~s})$ over which the sensor output is averaged at the beginning and end of each test, $\Delta F_{\text {drift }}$ is the change in sensor output due to drift and is estimated by using the slope of the sensor output at the beginning and end of each test, and $F_{\text {shear }}$ 
is the applied shear force for a given test.

Drift error (dynamic):

$$
\left[\begin{array}{c}
1 / n_{1} \sum_{n=N-n_{1}+1}^{n=N} F_{\text {sens }}(n \Delta t) \\
\frac{-\left(1 / n_{1}\right) \sum_{n=0}^{n=n_{1}-1} F_{\text {sens }}(n \Delta t)}{1 / n_{1}} \sum_{n=0}^{n=n_{1}-1} F_{\text {sens }}(n \Delta t)
\end{array}\right] \times 100
$$

where $n_{1} \Delta t$ is a chosen length of time $(0.25 \mathrm{~s})$ over which the sensor output is averaged at the beginning and end of the $10 \mathrm{~s}$ interval during which the dynamic drift error is considered.
Dynamic accuracy error:

$$
\left[\frac{\sqrt{\left(1 /\left(n_{2}-n_{1}+1\right)\right) \sum_{n=n_{1}}^{n=n_{2}}\left(\tilde{F}_{\text {sens }}(n \Delta t)\right)^{2}}}{-\sqrt{1 /\left(n_{2}-n_{1}+1\right) \sum_{n=n_{1}}^{n=n_{2}}\left(\tilde{F}_{\text {ref }}(n \Delta t)\right)^{2}}}\right] \times 100
$$

where $\tilde{F}_{\text {ref }}$ and $\tilde{F}_{\text {sens }}$ were high-pass filtered to remove the static load and low frequency drift from $F_{\text {ref }}$ and $F_{\text {sens }}$, and $n_{1}$ and $n_{2}$ are the first and last points in the data segment that was used (data segments were $4-8 \mathrm{~s}$ long, depending on sensor). 
